The Title of this Volume

Editors : will be set by the publisher

EAS Publications Series, Vol. ?, 2021

\title{
THREE DIMENSIONAL MOLECULAR LINE TRANSFER STUDY OF EXTRAGALACTIC ISM : AGN/STARBURST CONNECTION
}

\author{
Masako YAMADA $^{1}$
}

\begin{abstract}
Molecular gas in external galaxies is a subject of crucial importance for observational and theoretical studies of galaxy formation. Compact molecular gas around an active galactic nuclei (AGN) is expected to be an energy budget of AGN and/or the nuclear starburst. Recent observational studies suggest that line ratios in millimeter and submillimeter band may be a good tool to reveal the long-standing question on the origin of activity - AGN or nuclear starburst. We have constructed a powerful "telescope" of theory, three-dimensional nonLTE line transfer code, preceding the high resolution and sensitivity observations such as ALMA.
\end{abstract}

\section{Introduction}

Current progress in millimeter and submillimeter observations have found peculiar signature in ratios of molecular lines towards numbers of compact gas at the centers of active galaxies. The pioneering works of Kohno et al. (2001, 2005) showed that some of nearby Seyfert galaxies have enhanced ratio of $R_{\mathrm{HCN} / \mathrm{HCO}^{+}}$ as high as 2.5 or more. Following observations of various molecular lines have given rise of discussion of peculiar chemistry such as photon dominated region (PDR) or X-ray dominated region (XDR) (Meijerink et al. 2005, 2007, Aalto et al. 2007.) However, since the compact molecular core is not resolvable with current $\mathrm{mm} / \mathrm{submm}$ observational instruments, one has to take into account of internal structures within a core. Hydrodynamic simulations present highly inhomogeneous density and temperature structure within a compact molecular gas at the center of a galaxy (Wada \& Tomisaka 2005). In an inhomogeneous molecular gas naturally expected is the inhomogeneous population distribution. We performed three-dimensional non-thermodynamic equilibrium (nonLTE) molecular line transfer simulations, and examined the effects of excitation status inside a clumpy torus.

\footnotetext{
${ }^{1}$ ALMA Project Office, National Astronomical Observatory of Japan
} 
The Title of this Volume

\section{Ratio of $\mathrm{HCN}$ and $\mathrm{HCO}^{+}$Lines in Millimeter Band}

\subsection{Simulations}

We first performed hydrodynamic simulations of molecular gas in a steady gravitational potential of supermassive black hole of $M_{\mathrm{SMBH}}=10^{8} M_{\odot}$ and galactic halo (see for details, Wada \& Tomisaka 2005, Yamada et al., 2007b). The hydrodynamic simulation explicitly includes radiative cooling, feedback from supernovae, strong UV heating $\left(G_{0}=10\right)$ for thermal evolution as well. Resultant molecular ISM has a highly inhomogeneous structure in density, temperature, and turbulent velocity. Next we calculated three-dimensional nonLTE line transfer of $\mathrm{HCN}$ and $\mathrm{HCO}^{+}$pure rotational lines using a snapshot data of hydrodynamic simulation. We assumed spatially uniform molecular abundance distribution in order to examine the effect of clumpy excitation conditions on the line ratio $R_{\mathrm{HCN} / \mathrm{HCO}^{+}} \equiv I_{\mathrm{HCN}(1-0)} / I_{\mathrm{HCO}^{+}(1-0)}$ separately from chemistry models.

\subsection{Intensity Distribution and $R_{\mathrm{HCN} / \mathrm{HCO}^{+}}$}
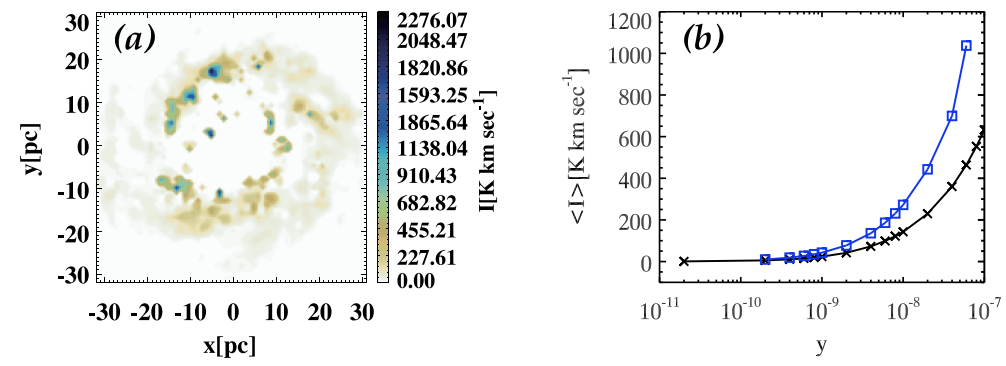

Fig. 1. Integrated intensity distribution of $\mathrm{HCN}(1-0)$ line of line transfer simulation with $y=2 \times 10^{-9}$ (left panel). Right panel shows integrated intensity averaged over the fieldof-view as a function of $y$. Line with squares denotes $\mathrm{HCO}^{+}(1-0)$ line, and line with crosses denots $\mathrm{HCN}(1-0)$ line, respectively.

In Figure 1 (a) we present integrated intensity distribution of $\mathrm{HCN}(1-0)\left(I_{\mathrm{HCN}}\right)$ line. The distribution of $I_{\mathrm{HCN}}$ shows clumpy structure, reflecting the highly inhomogeneous internal structure of the model torus. The typical size of the bright spots in Fig. 1 is $\approx 0.03^{\prime \prime}$ if the torus is located at the distance of $D=20$ Mpc. Thus though current mm-telescopes smear out these internal structures, high angular resolution of forthcoming telescope such as Atacama Large Millimeter/submillimeter Array (ALMA) will reveal inhomogeneous structures in molecular tori in distant galaxies. The Fig 1 (b) plots integrated intensities of $\mathrm{HCN}(1-0)$ and $\mathrm{HCO}^{+}(1-0)$ avraged over the "field of view" of our simulations as a function of fractional molecular abundance $y$. For the same value of $y,\left\langle I_{\mathrm{HCO}^{+}}\right\rangle$is always larger than $\left\langle I_{\mathrm{HCN}}\right\rangle$, which means that a ratio of $R_{\mathrm{HCN} / \mathrm{HCO}^{+}} \sim 2$ observed 
in some of nearby galaxies requires onverabundant $\mathrm{HCN}$ compared with $\mathrm{HCO}^{+}$ $\left(y_{\mathrm{HCN}} \approx 10 \times y_{\mathrm{HCO}^{+}}\right)$. This result and recent chemical studies of PDR and XDR do not seem to support the original idea of $\mathrm{mm} / \mathrm{submm}$ molecualr line diagnostics of AGN/starbust connection (e.g. Kohno et al. 2001). Other models, such as mid-InfraRed (MIR) photon pumping (e.g., Graciá-Carpio et al.2006 Aalto et al.2007) or hot core chemistry (e.g., Stäuber et al:2005) have been proposed, though, theoretical model description of molecular line ratios been controversial.

\section{Multi-Transition Analysis in Inhomogeneous Torus}

Current progress in submm observations enables multi-transion analysis and probe of warm and dense gas at the centers of external galaxies. In Figure 2 ratio of integrated intensities $\left\langle I_{\mathrm{HCN}(4-3)}\right\rangle$ and $\left\langle I_{\mathrm{HCN}(1-0)}\right\rangle$ (hereafter denoted as $R_{43 / 10}$ ) is plotted as a function of fractional molecular abundance $y$. In our simulation results ratio $R_{43 / 10}$ clearly decreases with $y$.
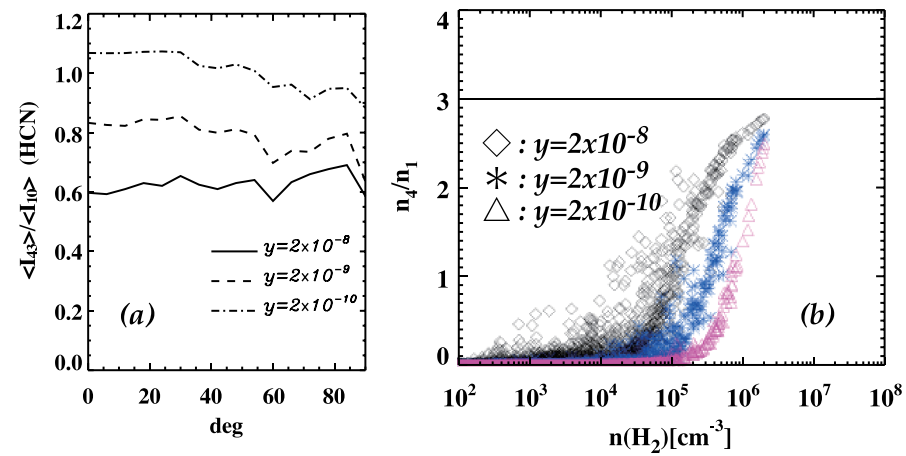

Fig. 2. $R_{43 / 10}$ as a function of viewing angle with respect to rotational axis of the torus. Right panel shows population ratio of $J=4$ and 1 , or excitation temperature of $T_{\mathrm{ex}}(41)$.

On the other hand, one-zone analysis predicts a reverse trend : if $y$ is small, optical thickness $\tau_{0}$ is expected to be small, then the ratio $R_{43 / 10}$ will be

$$
R_{43 / 10} \approx \frac{h \nu_{43} n_{4} A_{43} /\left(h \nu_{43}\right)^{2}}{h \nu_{10} n_{1} A_{10} /\left(h \nu_{10}\right)^{2}} \propto \frac{\sum_{J^{\prime} \geq 4} \gamma_{0 J^{\prime}}}{\sum_{J \geq 1} \gamma_{0 J}} \approx 0.3
$$

where $\gamma_{0 J}$ is collisional excitation constant from energy level $J=0$ to $J$. If $y$ is large, $\tau_{0}$ will be also large, and $R_{43 / 10}$ approaches unity.

This result indicates the importance of the inhomogeneity in structure of the torus. We model the clumpy torus as two-phase ISM consists of 1) dense clumps with $n \sim n_{\text {crit }}$ and 2) tenuous ambient medium with $n \ll n_{\text {crit. }}$. If we assume high kinetic temperature $\left(k_{B} T_{\text {kin }} \gg h \nu\right)$ and optically thin over a whole region, 
intensity ratios become (see eqs. [9] and [10] of Yamada et al. 2007a)

$$
\begin{aligned}
\left\langle R_{43 / 10}^{\prime}\right\rangle_{d} & \simeq \frac{\left\langle n_{4} A_{43} h \nu_{43}\right\rangle}{\left\langle n_{4} A_{43} h \nu_{43}\right\rangle} \sim \frac{4^{5}}{3}\left(\frac{n_{4}}{n_{1}}\right) \lesssim 1000, \\
\left\langle R_{43 / 10}^{\prime}\right\rangle_{t} & \simeq \frac{\left\langle h \nu_{43} \sum_{J \geq 4} \gamma_{0 J} n_{0}\right\rangle}{\left\langle h \nu_{10} \sum_{J \geq 1} \gamma_{0 J} n_{0}\right\rangle} \sim J=4 .
\end{aligned}
$$

Mean ratio $\left\langle R_{43 / 10}\right\rangle_{\text {all }}$ in a synthetic observation beam can be written as a kind of average of $\left\langle R_{43 / 10}\right\rangle_{d}$ and $\left\langle R_{43 / 10}\right\rangle_{t}$,

$$
\left\langle R_{43 / 10}^{\prime}\right\rangle_{\text {all }}=\eta\left\langle R_{43 / 10}^{\prime}\right\rangle_{d}+(1-\eta)\left\langle R_{43 / 10}^{\prime}\right\rangle_{t},
$$

where $\eta$ denotes a volume filling factor of dense clumps. Equation (3.4) means that if $\eta$ is sufficiently smaller than unity, $R_{43 / 10}$ can take a value of order unity even if the medium is optically thin. In our radiative transfer simulations, "dense clumps" can safely be defined as regions with $n \geq 10^{5} \mathrm{~cm}^{-3}$ (close to $n_{\text {crit }}$ of HCN(1-0) transition), and $\eta_{\text {sim }}$ is indeed $\approx 10^{-3}$. Furthermore, nonLTE population (occasional $\tau_{0}<0$ for $J=1-0$ transitions) and inhomogeneous optical thickness distribution complicate the $R_{43 / 10}$ distributions (see right panel of Fig. 2). Our analysis demonstrates the importance of inhomogeneity of molecular gas in estimation of physical properties from line ratios from unresolved observational data. Inherent inhomogeneity should be paid much attention when interpreting line ratio in terms of chemical evolution of molecular gas.

\section{Summary}

We examine molecular line diagnostics of molecular gas in the center of external galaxies using three dimensional radiative transfer simulations. Our results demonstrate that realistic inhomogeneous molecular gas can produce line ratio values unexpected from one-zone analysis, and emphasize the ability and applicability of our approach, that is, compilation of high-resolution hydrodynamic simulations and three-dimensional radiative transfer calculations ("telescope of theory") towards forthcoming ALMA era.

\section{References}

Aalto, S. et al. 2007, A\&A, 464, 193.

Graciá-Carpio, J. et al. 2006, ApJ, 640, L135.

Kohno, K. et al. 2001, ASP Conference Proceedings, 249, 672.

Kohno, K. 2005, AIP Conference Proceedings, 783, 203.

Meijerink, R. \& Spaans, M. 2005, A\&A, 436, 397.

Meijerink, R., Spaans, M. \& Israel, F. P. 2007, A\&A, 461, 793.

Stäuber et al. 2005, A\&A, 440, 949.

Yamada, M. et al. 2007, ApJ, 657, 849.

Yamada, M., Wada, K., \& Tomisaka, K. 2007, ApJ, 671, 73.

Wada, K. \& Tomisaka, K. 2005, ApJ, 619, 93. 